\title{
Curcumin combining with si-MALAT1 inhibits the invasion and migration of colon cancer $S W 480$ cells
}

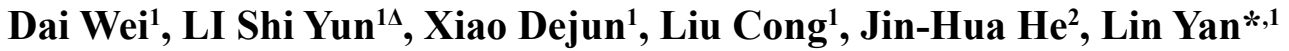 \\ ${ }^{1}$ Department of Clinical Laboratory, Gangzhou People's Hospital, Ganzhou, China, ${ }^{2}$ Department of Laboratory Medicine, \\ Central Hospital of Panyu District, Guangzhou, Guangdong, China
}

\begin{abstract}
To study the effect of small interfering RNA targeting metastasis-associated lung adenocarcinoma transcript1 (si-MALAT1) combining with curcumin on the invasion and migration abilities of human colon cancer SW480 cells, and to explore the involved molecular mechanism. The recombinant lentiviral vector expressing si-MALAT1 was constructed, and its titer was determined by gradient dilution method. The colon cancer SW480 cells with stable expression of si-MALAT1 was established, followed by treatment with curcumin at different concentrations. The effect of curcumin or si-MALAT1 alone and the combination of the two on the cell activity was detected by MTT assay. The cell invasion and migration abilities were detected by transwell and scratch-wound assay. The relative expression level of MALAT1 was detected by RT-qPCR. The protein expression was determined by Western blot analysis. The IC50 of curcumin alone was $77.69 \mu \mathrm{mol} / \mathrm{L}$, which was $51.17 \mathrm{~mol} / \mathrm{L}$ when combined with curcumin and random sequence. The IC50 of curcumin was $30.02 \mu \mathrm{mol} / \mathrm{L}$ when combined with si-MALAT1. The increased susceptibility multiples was 2.58 . The wound healing rates were $30.9 \%$ and $67.5 \%$ after treatment with si-MALAT1 combined with curcumin for $24 \mathrm{hrs}$ and $48 \mathrm{hrs}$, respectively. The numbers of invasion cells were $200 \pm 12,162 \pm 13,66 \pm 8,53 \pm 4$ and $16 \pm 3$ after treatment with si-MALAT 1 combined with curcumin for $48 \mathrm{hrs}$. The relative expression level of lncRNA-MALAT1 in the curcumin group was $68 \%$, and the relative expression level of lncRNA-MALAT 1 in si-MALAT1group was $56 \%$, and that for the combination treatment group was about $21 \%$. The protein expression levels of $\beta$-catenin, c-myc and cyclinD1 were significantly down-regulated upon treatment with certain concentration of si-MALAT1 alone or combined with curcumin.si-MALAT1 could significantly inhibit the invasion and migration of SW480 cells by enhancing the sensitivity of SW480 cells to curcumin. The mechanism involved mignt be related to the down-regulation of $\beta$-catenin, c-myc and cyclinD1 proteins.
\end{abstract}

Keywords: Curcumin/ pharmacology. LncRNA-MALAT1. RNA, Small Interfering/ drug effects. Colon cancer. Invasion. Migration. Colonic Neoplasms/ prevention \& control. Colonic Neoplasms/ drug therapy. Neoplasm Invasiveness/ prevention \& control. Cell Migration Inhibition/ drug effects.

\section{INTRODUCTION}

Colon cancer is the third most common malignant tumor in the world, and its morbidity and mortality are increasing year by year. Patient received post-operation adjuvant chemotherapy is prone to death due to metastasis and recurrence (Torre et al., 2015; Burt, 2013). Therefore, it is urgent to clarify its pathogenesis and improve the therapeutic effect.

\footnotetext{
*Correspondence: Y. Lin. Department of Clinical Laboratory, Gangzhou People's Hospital, Ganzhou 341000, China. Telephone: 86-0797-5889135. E-mail:2529093393@qq.com (iD

${ }^{\Delta}$ Has equal contribution
}

Long-chain non-coding RNA (lncRNA) is a type of non-coding RNAs with a length of more than 200 nucleotides. It is involved in many physiological and pathological processes, and considered to be closely related to the occurrence and development of tumor. They can also play roles in both tumor suppression and cancer promotion. However, the study of the molecular mechanism of $\operatorname{lncRNA}$ regulation is still at a preliminary stage (Fatima et al., 2015). Studies have shown that the abnormal expression of lncRNA was associated with the growth and invasion of colon cancer cells (MA et al., 2016). Metastasis-related lung adenocarcinoma transcript 1 (Metastasis-associated lung adenocarcinoma transcript 1, MALAT1) is widely expressed in human 
normal tissue, especially in pancreas and lung tissues (Zheng et al., 2014). It is also found to be highly expressed in colon cancer tissues. Its expression and neural infiltration could affect the invasion and migration of colon cancer cells, and therefore were considered as two independent risk factors for the poor prognosis of colon cancer patients (Ji et al., 2014).

Compared to the current conventional treatment strategy for cancer, traditional Chinese medicine has the advantages of low toxicity and being able to improve the patient's immunity. It will have unparalleled advantages in cancer prevention, early cancer treatment, and even in the application of middle and late stage cancer. Curcumin, a diketone compound, a substance derived from the family Curcumin. It has been shown to have anti-inflammation, anti-oxidation and anti-tumor effects, as well as reducing blood lipids (Anand et al., 2008). Curcumin was also found to be able to inhibit invasion and metastasis of colon cancer cells, but the molecular mechanism involved is still not clear (Kunnumakkara, Anand, Aggarwal, 2008).

In this study, small interfering RNAs (siRNAs) were used to reduce the level of MALAT1 expression in colon cancer SW480 cells, which were further treated with curcumin. We aimed to determine the effect of this combination treatment on the viability and migration of colon cancer SW480 cells, and further clarify the potential molecular regulatory mechanism involved. It will provide the theoretical basis for the clinical treatment of colon cancer using integrated Chinese and western medicine.

\section{MATERIAL AND METHODS}

\section{Material}

Liposome LipofectamineTM2000 was purchased from Invitrogen Company; DMEM/F12 medium was purchased from Hyclone Company; RIPA cell lysate (Shanghai Sheneng betting Biotechnology Co., Ltd.); BCA protein concentration determination kit (Beijing Boosen Biotechnology Co., Ltd.). MTT reagent from the Japanese Institute of Tongren Chemistry; RNA extraction reagent TRIzol (Invitrogen Company; ECL kit (Biyuntian Biotechnology Research Institute); Polyclonal resistance to $\beta$-catenin $\mathrm{c}$ myc and $\mathrm{c}$ YclinD1 antibody, monoclonal GAPDH antibody (Cell Signaling, USA); Curcumin was purchased from Sigma Company of USA and dissolved into $10 \mathrm{mmol} / \mathrm{L}$ standby with DMSO. The recombinant lentivirus plasmid pLVX-shRNA2 and the packaging plasmid PLD2 were obtained From Shanghai Jima Co., L). And encapsulated plasmid PLD3 is provided by Shanghai Jima Co., L.

\section{Cell culture}

Colon cancer SW480 cells were purchased from Shanghai Cell Bank Center. The cells were cultured in DMEM / F12 contained 10\% newborn bovine serum at $37^{\circ} \mathrm{C}$ in the incubator containing $5 \% \mathrm{CO}_{2}$. Cells was digested and subcultured with $0.25 \%$ trypsin every 2-3 days. Cells with trypan blue rejection rate $>95 \%$ in logarithmic growth period were used for further experimentation.

\section{Construction of colon cancer SW480 cells stably expressing siRNA-MALAT 1}

The sense chain of siRNA sequence of MALAT1 was 5'-GAGGUGUAAAGguUUAUTT-3'. The antisense chain was 5-AUA-AAAUCCCUUU ACACCUCTT-3; The sense chain of random sequence was 5'-UUCUCCGAACGUGUC ACGUTT-3'. The antisense chain was 5-ACGUGACACGUCGGAGAATT-3; the construction of siRNA-MALAT 1 expressing lentiviral vector was conducted by Shanghai Jima Limited Technology Co., Ltd. Recombinant lentivirus plasmids, packaging plasmids PLD2 and encapsulated plasmids PLD3 were co-transfected into $293 \mathrm{~T}$ cells. $24 \mathrm{hrs}$ after the transfection, $10 \mathrm{~mL}$ fresh complete culture medium (containing $1 \%$ pairs of antibodies) was replaced. The virus-containing supernatant was then collected after 48 hrs, and then continuously diluted with a gradient of 10 times on a 48 -well cell culture plate. The $10 \mu \mathrm{L}$ of the diluted virus-containing supernatant was then added to the cell culture. After 48 hours of cultivation, the collected supernatant was diluted. The fluorescent cells were counted under inverted fluorescence microscope. The titer of the virus was defined by transducing units (TU). The virus titer was calculated according to the following formula: $(\mathrm{Tu} / \mathrm{m})=($ green fluorescent cell number / field of vision $) \times($ visual field number $/$ hole number $) \times$ virus dilution multiple / virus volume. The SW480 cells were then infected with packaged lentivirus. The SW480 cell lines expressing stably siRNA-MALAT1 were established according to the instructions.

\section{MTT assay}

SW480 cells in logarithmic growth phase were cultured in serum-free DMEM/F12 medium. The cell concentration was adjusted to $1 \times 10^{5}$ cells $/ \mathrm{mL}$, and then $100 \mu \mathrm{L}$ per well of the cell suspension inoculated in a 96-well plate. The cells were divided into two groups: blank control group (no treatment);si-MALAT1 group 
(treated with $10 \mu \mathrm{mol} / \mathrm{L}$ of si-MALAT1); si-MALAT1 combined with curcumin group (treated with $15 \mu \mathrm{mol} / \mathrm{L}$ of si-MALAT1 and curcumin); Negative control group (treating with $15 \mu \mathrm{mol} / \mathrm{L}$ of random sequence) and the Curcumin group (treated with 5, 10,20,40 and $80 \mu \mathrm{mol} / \mathrm{L}$ of curcumin); after $48 \mathrm{hrs}$ of cultivation, $20 \mu \mathrm{L}$ of MTT solution was added to each well. After $4 \mathrm{hrs}$, the culture medium was carefully discarded, and $150 \mu \mathrm{L}$ DMSO was added to each well. The resultant violet crystal was completely dissolved by oscillating at low speed for $10 \mathrm{~min}$. The absorbance of each well was measured at the wavelength of $490 \mathrm{~nm}$ and the average value was obtained.

\section{Transwell assay}

The Matrigel was diluted in serum-free DMEM/ F12 medium and then added into the transwell chamber. containing $8 \mu \mathrm{m}$ small polycarbonate filter membrane. $5 \times 10^{5}$ of SW480 cells were diluted in $100 \mu \mathrm{L}$ serumfree medium, and $600 \mu \mathrm{L}$ of the DMEM/F12 medium containing $10 \%$ serum was added in the lower chamber. Each group was cultured for $24 \mathrm{hrs}$ in triplicate at $37^{\circ} \mathrm{C}$ in the incubator containing $5 \% \mathrm{CO}_{2}$. Cells in the culture chamber was then removed and fixed with methanol for 20 mins, followed by staining with $1 \%$ crystal violet for 20 mins. The matrix glue and the non-invasive cells at the bottom of the upper chamber were gently wiped off with wet cotton swabs, then the chamber was inverted. The number of cells passing through the membrane was counted under the 100x optical microscope.

\section{Scratch-wound assay}

$5 \times 10^{5}$ of SW480 cells were added to each well of a 12 -well plate and allowed to grow until a confluent monolayer was formed. Then the cell monolayer was scraped in a straight line to create a gap with a pipet tip. The cell debris was then removed by washing with $1 \times$ PBS for 3 times. Culture medium was then added again and cells were allowed to grow for $48 \mathrm{hrs}$ before taking the microscopy imagines. The distance between one side of scratch and the other was measured using Image $J$ software.

\section{Detection of relative expression level of MALAT1 by RT-qPCR}

M-MLV Reverse Transcriptase was used for the reverse transcription of mRNA to complementary DNA (cDNA), which was later used as the PCR template. Each experiment was performed in triplicate. The expression levels of U6 and U6 was used as an internal control for mRNAs. The sense chain sequence of primer. MALAT1 was 5'-AGGCGTTGTGGTAGAGA-3'. The sequence of antisense chain is 5 GGATTTACCAACCACTCGC-3; The sense chain sequence of U6 was 5'-CTCGCTT CGGCAGCACA3'. The antisense sequence was 5 AACGCTTCACGAATTTGCGT-3. Using U6 as internal reference, the relative expression of MALAT1 was calculated.

\section{Western blot analysis}

The cell was lysed in RIPA buffer containing a proteinase inhibitor cocktail (Biocolor BioScience \& Technology, Shanghai, China). After centrifugation at $4{ }^{\circ} \mathrm{C}$ for 15 mins, the pellets were discarded. The protein concentration of each group was determined by bicinchoninic acid (BCA) Protein Assay Kit (Bioss, Beijing, China). The protein was then separated on the sodium dodecyl sulfate-polyacrylamide gel electrophoresis (SDS-PAGE) and electrotransferred to PVDF membranes. GAPDH was used as the internal reference. The ratios of $\beta$-catenin,c-myc,cyclinD and GAPDH were calculated and interpreted as the relative expressions of $\beta$-catenin,c-myc and cyclinD1 proteins. All experiments were repeated for three times (absolute gray value = gray value - background gray value).

\section{Statistical analysis}

All the results were represented the average of at least three independent experiments. Data were expressed as the mean \pm SD and analyzed by one-way analysis of variance (ANOVA) and Student's $t$ test. A $p<0.05$ was considered statistically significant.

\section{RESULTS}

\section{The lentiviral vector expressing si-MALAT1 was successfully constructed}

The recombinant lentiviral plasmid, packaging plasmid PLD2 and the envelope plasmid PLD3 were co-transfected into $293 \mathrm{~T}$ cells. The virus-containning supernatant was then collected at $72 \mathrm{~h}$, which viral titer was $1 \times 109 \mathrm{TU} / \mathrm{mL}$ determined using 10 times continuous gradient method(Figure 1A). The GFP virus expression was observed by fluorescence microscope (Figure 1B). Sequencing results of the recombinant plasmid products were completely compatible with the designed primer sequences using the Blast ratio. It suggested that the 
lentivirus expression vector was successfully constructed (Figure 1C).

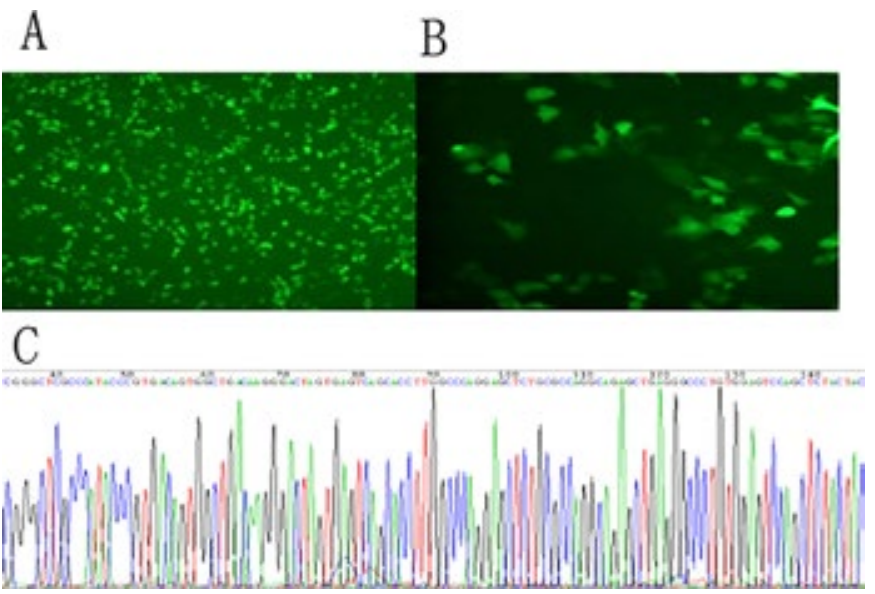

FIGURE 1 - Determination of lentivirus titer and identification of recombinant plasmid products by sequencing. A: The recombinant plasmid expressing si-MALAT1 was observed under fluorescence microscope (40x); B: The plasmid expressing empty vector was observed under fluorescence microscope; $C$ : Identification of recombinant plasmid products by sequencing .

\section{Curcumin combined with si-MALAT1 inhibited the SW480 cell viability}

The IC50 of curcumin alone was $77.69 \mathrm{umol} \cdot \mathrm{L}^{-1}$. The IC50 of curcumin combined with random sequence was $51.17 \mathrm{umol} \cdot \mathrm{L}^{-1}$; The IC50 of curcumin combined with si-MALAT1 mimics was $30.02 \mathrm{umol} \cdot \mathrm{L}^{-1}$. The results showed that curcumin combined with si-MALAT1 could significantly inhibit the viability of SW480 cells (Figure 2).

\section{Curcumin combined with si-MALAT 1 inhibited the migration of SW480 cell}

SW480 cells were treated with certain concentration of si-MALAT1 mimics, random sequences, curcumin and si-MALAT1mimics on for $24 \mathrm{~h}$ or $48 \mathrm{~h}$. The wound healing rate of the curcumin group alone was $30.9 \%$ and $67.5 \%$ after treatment for $24 \mathrm{~h}$ and $48 \mathrm{~h}$, respectively. The wound healing rate of si-MALAT1 mimics combined with curcumin group was $15.75 \%$ and $24.8 \%$ after treatment for $24 \mathrm{~h}$ and $48 \mathrm{~h}$, respectively. The si-MALAT1 analogue combined with curcumin group had significantly lower wound healing rate than those of the blank control group and the negative control group $(P<0.05)$. There was no significant difference between the negative control group and the blank control group $(P>0.05)$. The results suggested that Si-MALAT1 combined with curcumin

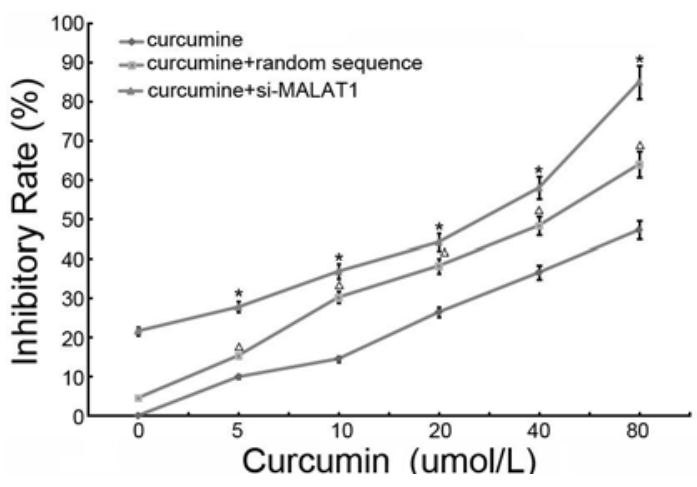

FIGURE 2 - Cell inhibition rates in the curcumin group, the curcumin and random sequence group, and the curcumin and si-MALAT1 group $* P<0.05, \triangle P>0.05, \mathrm{n}=3$.

could significantly inhibit the migration of SW480 cells. (Figures $3 \mathrm{~A}$ and $\mathrm{B}$ )

\section{Curcumin combined with si-MALAT1 inhibited the invasion of SW480 cell}

Result from the transwell assay showed that, the number of invasion cells for the blank control group, the negative control group, the curcumin group, and the curcumin combined with si-MALAT1 mimics group were $200 \pm 12,162 \pm 13,66 \pm 8,53 \pm 4$ and $16 \pm 3$, respectively. The number of invasion cells in the curcumin combined with si-MALAT1 mimics group was significantly lower than those in other groups $(P<0.05)$. It suggested that si-MALAT1 combined with curcumin could inhibit the invasion of SW480 cells (Figure 4).

\section{Curcumin combined with si-MALAT 1 inhibited the expression of MALAT1}

SW480 cells were treated with curcumin alone or combined with si-MATAT 1 at a certain concentration for $48 \mathrm{hrs}$, and the expression of IncRNA-MALAT1 was then detected by real-time quantitative PCR. The results showed that the relative expression levels of lncRNAMALAT 1 were $68 \%, 56 \%$ and $21 \%$ in the curcumin group, the si-MALAT1 group, and the combination treatment group, respectively. The difference between the combination treatment group and the single treatment group was statistically significant $(P<0.05)$ (Figure 5).

\section{Curcumin combined with si-MALAT 1 down- regulated the protein expression of $\mathrm{c}-\mathrm{myc}$, cyclinD 1 and $\beta$-catenin}

si-MALAT1 was transfected into SW480 cells for $48 \mathrm{~h}$. The results of Western blotting showed that the 

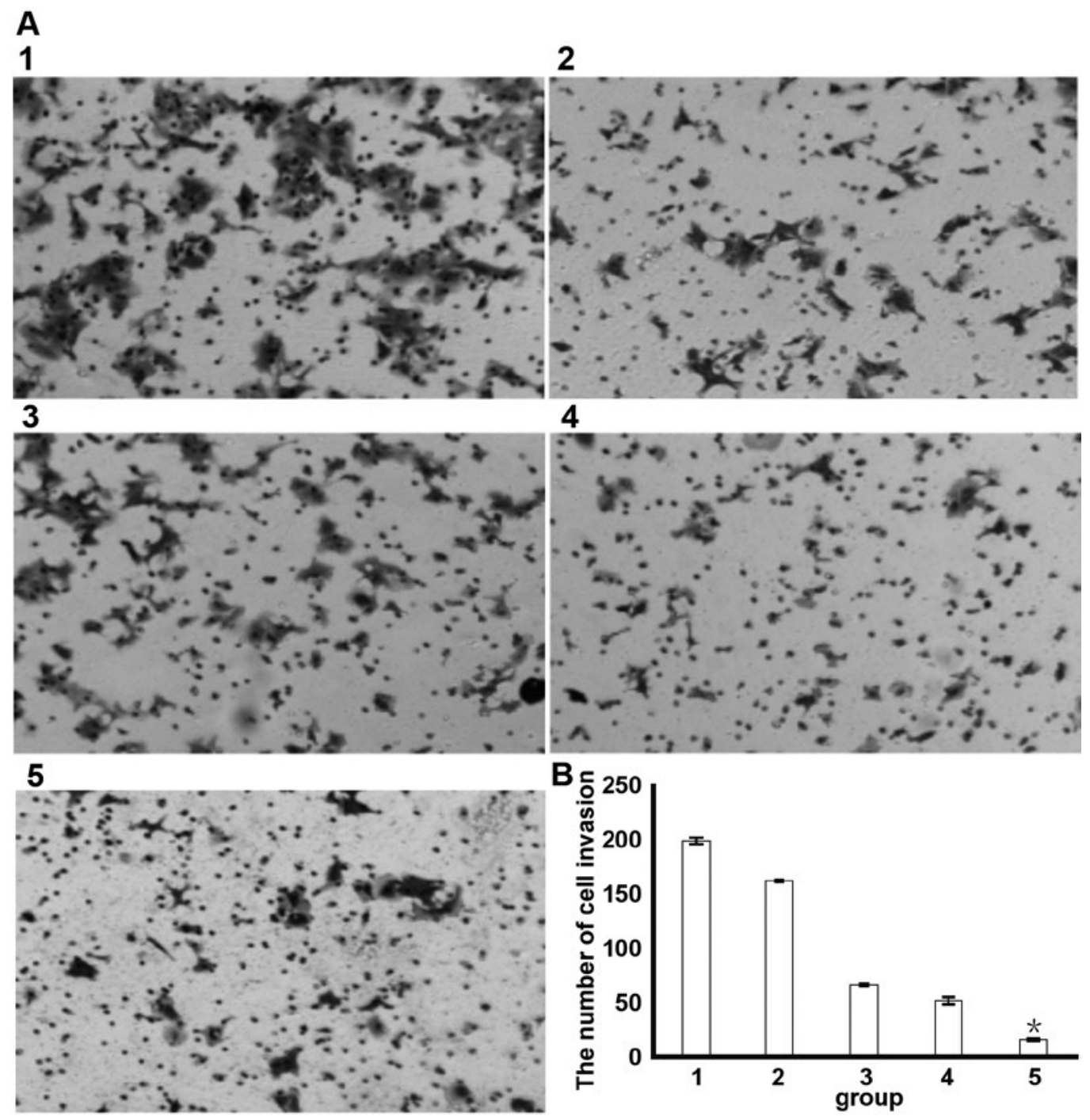

FIGURE 3 - The migration ability of SW480 cells was detected by transwell assay. A: The cell migration distance of each group after treatment for $24 \mathrm{~h}$ or $48 \mathrm{~h}$ was observed under fluorescence microscope (40X); C: The quantitative analysis of of the wound healing rates in each group after treatment for $48 \mathrm{hrs}$. Mean \pm SD. $n=3$. $* P<0.05, * * P<0.05$.

protein expression levels of $\beta$-catenin, c-myc and cyclinD1 were significantly down-regulated when treatment with certain concentration of si-MALAT1 mimics alone or combined with curcumin (Figure 6).

\section{DISCUSSION}

Colon cancer is one of the most common malignant tumors of digestive tract in the world, which is seriously threatening human life and health. The occurrence and development of colon cancer is a multi-step process. The mortality rate of colon cancer in China is as high as $60 \%$. The conventional treatment for early colorectal cancer is surgical resection. The 5-year survival rate can reach $90 \%$ to $95 \%$. The metastasis and invasion of colon cancer are the main causes of postoperative recurrence and the death of patients (Wu et al., 2014). Therefore, clarifying the molecular mechanism involved in the invasion and metastasis of colon cancer cells will shed the light on the development of effective therapeutic treatment strategy for colon cancer, and hopefully lower its mortality rate.

The differential expression of IncRNA in colon cancer is closely related to clinical features, suggesting that lncRNA is involved in the invasion and metastasis of colon cancer (Chen et al., 2016). Some studies have shown that lncRNA-MALAT1 was highly expressed in colon cancer tissues, and high expression of lncRNAMALAT1 was associated with poor prognosis and lower chemotherapeutic tolerance (Mercer, Dinger, Mattick, 2009). Curcumin is a type of polyphenols extracted from the rhizomes of turmeric and other plants. It has a wide range of pharmacological usage. It has been shown that 

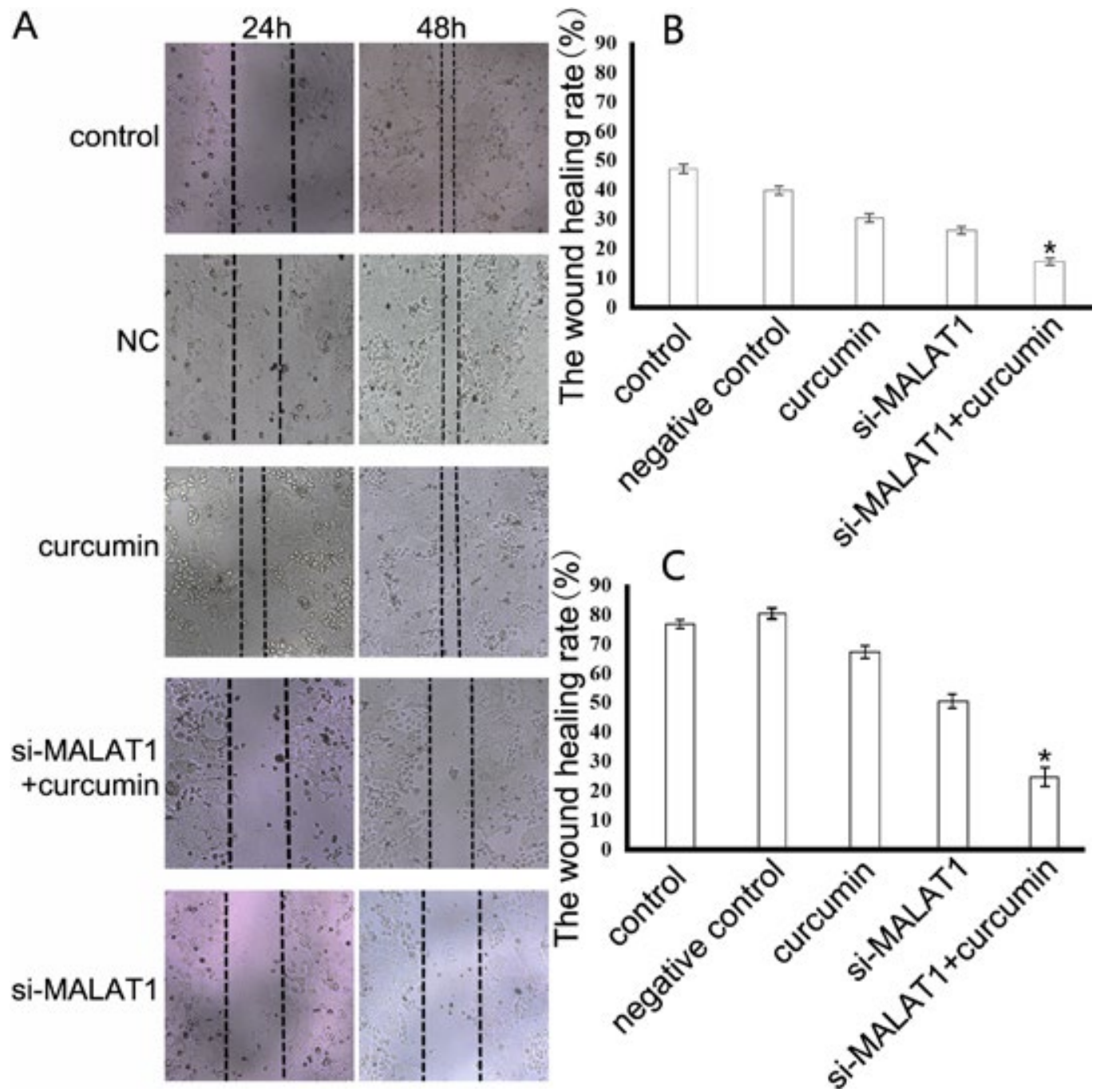

FIGURE 4 - The invasion ability of SW480 cells in different groups was evaluated by scratch-wound assay. A: The invasion cells in each group were observed under fluorescence microscope (40X; B: The quantitative analysis of the invasion cells in each group: 1: Control group; 2: Negative control group; 3: Curcumin group; 4: si-MALAT1 group; 5: Curcumin combined with si-MALAT1 group. Mean \pm SD. $n=3 . * P<0.05 v s$ other groups.

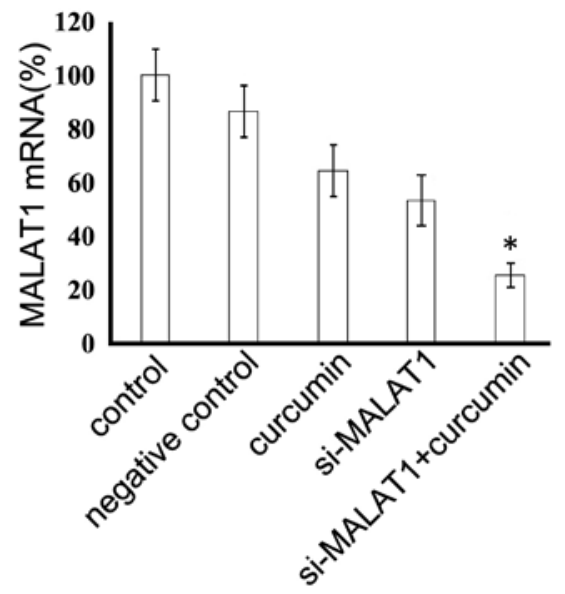

FIGURE 5 - The effect of curcumin on the expression of lncRNA-MALAT1 in the human colon cancer SW480 cells. Mean \pm SD. $n=3 . * P<0.05$ vs other groups. curcumin could inhibit metastasis and induce apoptosis in many kinds of tumors (Zhou et al., 2014).

In this study, the small interfering RNA sequence for MALAT1 was designed, and the recombinant lentivirus plasmid pLVX-shRNA2 was ligated with the encapsulated plasmid. The recombinant lentivirus plasmid si-MALAT1 was constructed, and used for infecting SW480 cells to establish a stable cell line expressing si-MALAT1. At the same time, the combination of si-MALAT1 with different concentrations of curcumin could significantly increase the sensitivity of SW480 cells to curcumin after treatment for $48 \mathrm{hrs}$. Curcumin combined with si-MALAT1 could inhibit migration and invasion of SW480 cells.

Wnt signaling pathway is not only closely related to embryonic development, but also contributes to human tumorigenesis (Heintze et al., 2017). $\beta$-catenin avoids 

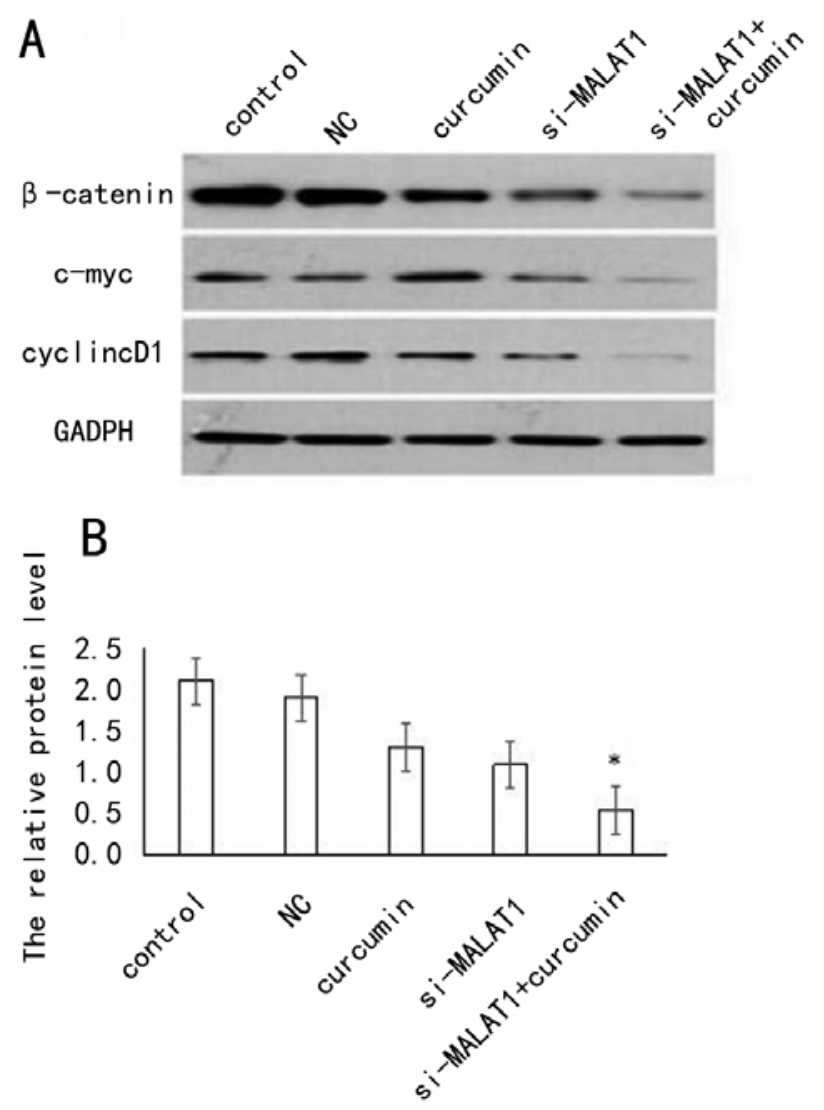

FIGURE 6 - The relative protein expression levels of $\beta$-catenin, c-myc and cyclinD1 detected by Western-blotting. A: $\beta$-catenin, c-myc and cyclinD1 expression in different treatment groups; $\mathrm{B}$ :. The relative protein expression levels of catenin, c-myc and cyclinD1 in different treatment groups, Mean \pm SD. $n=3$.* $P<0.05$ vs other groups.

degradation through ubiquitin proteasome pathway and enters nucleus aggregation to activate downstream target genes such as c-myc. Expression of cyclin D1 and VEGF can promote tumor occurrence and metastasis (Bienz, Clevers, 2000). In this study, 48 hrs after treatment with curcumin and si-MALAT1, the expression of MALAT1 in SW480 cells was down-regulated. At the same time, the protein expression of cyclin D1, $\beta$-catenin and c-myc was also decreased. The results suggested that curcumin combined with si-MALAT1 could inhibit the proliferation of SW480 cells. Migration and invasion of SW480 cells were achieved by down-regulating MALAT1 and downregulating cyclin D1.

$\beta$-catenin and c-myc in the Wnt signaling pathway .Curcumin could obviously suppress the growth of SW620 in colon cancer cells in vitro and in vivo through inhibiting the Wnt signaling pathway by up-regulating the expression of NKD2 gene, and then, down regulating expression of CXCR4 in tumor cells, which further inhibited the invasion and metastasis of tumor cells (Akira et al., 2000).

\section{CONCLUSIONS}

In conclusion, this study demonstrated in vitro that, curcumin combined with si-MALAT1 inhibited the viability, migration and invasion of SW480 cells by downregulating the expression MALAT1. And down-regulating the expression of $\beta$-catenin, c-myc and cyclin D1 in Wnt signaling pathway. However, the in vivo effect of curcumin needs to be further elucidated. The present study provides the theoretical basis for the clinical treatment of colon cancer using integrated Chinese and western medicine, and will hopefully aid the development of new therapeutic treatment strategy for colon cancer in the near future.

\section{ACKNOWLEDGEMENTS}

The present study was supported by Guiding Science and Technology Project of Ganzhou City, Jiangxi Province (Grant number: GZ2015ZSF105).

\section{REFERENCES}

Akira K. Regulation of cateinin signaling in the Wnt pathway. Biochemical and biophysical research communication 2000;268(2)243-248.

Anand P, Sundaram C, Jhurani S, Kunnumakkara AB, Aggarwal BB. Curcumin and cancer: an "old-age" disease with an "ageold" solution. Cancer Lett. 2008;267(1):133-64.

Bienz M, Clevers H. Linking colorectal cancer to Wnt signaling. Cell. 2000;103(2):311-20.

Burt RW. Colon cancer screening continues as pivotal to cancer prevention. J Natl Compr Canc Netw. 2013;11(12):1457-8.

Chen HL, Chen HT, Xu C, et al. The effects of curcumin on expression of NKD2 and CXCR4 genes in SW620 colon cancer cell. China Cancer. 2016;25(4):297-302.

Fatima R, Akhade VS, Pal D, Rao SM. Long noncoding RNAs in development and cancer: potential biomarkers and therapeutic targets. Mol Cell Ther. 2015;3(1):1-19.

Heintze JM. Developmental biology: renewal of NPCs requires MYC and $\beta$-catenin. Nat Rev Nephrol. 2017;13(12):723. 
Ji Q, Zhang L. Liu X, Zhou L, Wang W, Han Z, et al. Long noncoding RNA MALAT1 promots tumor growth and metastasis in colorectal cancer through binding to SEPQ and releasing oncogene PTBP2 fron SF-PQ/PTBP2 complex. Br J Cancer. 2014;111(4):736-48.

Kunnumakkara AB, Anand P, Aggarwal BB. Curcumin inhibits proliferation, invasion, angiogenesis and metastasis of different cancers through interaction with multiple cell signaling proteins. Cancer Lett. 2008;269(2):199-225.

Ma Y, Yang Y, Wang F, Moyer MP, Wei Q, Zhang P, et al. Long non-coding RNA CCAL regulates colorectal cancer progression by activating $\mathrm{Wnt} / \beta$-catenin signalling pathway via suppression of activator protein $2 \alpha$. Gut, 2016, 65(9):1494-504.

Mercer TR, Dinger ME, Mattick JS. Long non-coding RNAs: insights into functions. Nat Rev Genet. 2009;10(3):155-9.
Torre LA, Bray F, Siegel RL, Ferlay J, Lortet-Tieulent J, Jemal A. Global cancer statistics, 2012. CA Cancer J Clin. 2015;65(2):87-108.

Wu HJ, Liu HC, Chang YT, Lin S-R, Chang, L-S. Predictive biomarkers for the efficacy of concurrent chemoradiotherapy for patients with colorectal cancer. Biomarkers Genomic Med. 2014;6(4):163-6.

Zheng HT, Shi DB, Wang YW, Li XX, Xu Y, Tripathi P, et al. High expression of lncRNA MALAT1 suggests a biomarker of poor prognosis in colorectal cancer. Int J Clin Exp Pathol. 2014;7(6):3174-81.

Zhou DY, Ding N, Van Doren J, Wei XC, Du ZY, Conney AH. Effects of curcumin analogues for inhibiting human prostate cancer cells and the growth of human PC-3 prostate xenografts in immunodeficient mice. Biol Pharm Bull. 2014;37(6):1029-34.

Received for publication on $13^{\text {th }}$ April 2018 Accepted for publication on $16^{\text {th }}$ May 2018 